\title{
VERTEBRATE WILDLIFE DIVERSITY OF SREEPUR UPAZILA, MAGURA BANGLADESH
}

\author{
Mandal, A. K., M. F. Jaman ${ }^{1}$, M. M. Alam ${ }^{1}$, M. F. Rabbe ${ }^{1}$ and A. R. Shome ${ }^{1}$ \\ Govt. H.S.S. College, Magura, Bangladesh; ${ }^{1}$ Department of Zoology, Dhaka University, Dhaka-1000, \\ Bangladesh
}

\begin{abstract}
The species diversity and abundance of wildlife are the important indicators of a healthy ecosystem. A survey-based scientific study on species diversity, abundance and status of vertebrate wildlife was conducted from May 2015 to April 2016 at Sreepur upazila, Magura, Bangladesh. A total of 123 species of vertebrate wildlife was recorded during the 12 months of study period. Among them, eight species (6.5\%) were amphibians, $13(10.57 \%)$ reptiles, $84(68.29 \%)$ birds and 18 species (14.64\%) were mammals. Out of 84 species of birds, 45 (53.57\%) were passerines and 39 (46.43\%) non-passerines. Most of the observed birds (72 species, $85.72 \%$ ) were resident and the rest 12 species $(14.28 \%)$ were migratory. The highest number of wildlife species was observed in winter (108 species, $87.80 \%)$, particularly in December (62 species, 50.40\%). The lowest number of species was recorded in June (33 species, 26.83\%). Out of 123 species of vertebrate wildlife, three $(2.44 \%)$ were very common, nine $(7.32 \%)$ common, $26(21.14 \%)$ fairly common and 85 $(69.1 \%)$ were common. Of the total species, $117(95.12 \%)$ species were least concern, five (4.06\%) near threatened and one $(0.81 \%)$ was endangered according to IUCN Bangladesh 2015. Implementation of conservation and management plan is required to save the wildlife in the study area.
\end{abstract}

Key words: Richness; Abundance; Habitat; Threats; Conservation.

\section{INTRODUCTION}

Bangladesh acts as an intermediate area for the flora and fauna of the subcontinent due to its location (Khan 2018). The variation in climate (e.g. temperature, rainfall) and abiotic factors (e.g. soil and hydrology) has graded the whole country into 25 bio-ecological zones with diverse ecosystems (Nishat et al. 2002). These diverse ecosystems support more than thousands of wild fauna (AmphibiaMammalia) and some new species has been described recently (IUCN BD 2015, Shome et al. 2020b, Khan 2018, Al-Razi et al. 2020a and b, Hakim et al. 2020). At present, the country has a total of 127 mammals, 680 birds, 167 reptiles and 57 amphibian species (IUCN BD 2015).

The rich and diverse wildlife in Bangladesh have been playing important roles in ecological, cultural, economic, social sector. In agricultural sector, wildlife plays an important role in pest control (both vertebrate and invertebrate pest). Besides, diseases control, scavenging, pollinating, seed dispersal, mosquito controls and providing food to human were also done by them (Islam et al. 2018, Jaman et al. 1999, Mukul 2008). But, anthropogenic activities, habitat loss and indiscriminate killing are becoming the major causes of rapid population declining in this country (Khan 2018). The wildlife population decline and species extinction are related and can occur both in protected and non-protected areas.

Non-protected areas such as rural areas of Bangladesh hold a good number of habitats (e.g. haor, baor, canal, beel, pond, riverine islands, agricultural lands, fallow lands, homestead forest(s) gardens, roadside trees etc.) provide support for wildlife (IUCN BD 2015, Shome et al. 2020b, Khan 2018, Mukul 2008). Some researches on wildlife were conducted in different regions of Bangladesh (Jaman et al. 2021, Shome et al. 2020b, Islam et al. 2018, Hussain and Sarker 1997, Sarker et al. 2001, Hossain et al. 2004, Jaman et al. 2011, Rahman et al. 2011, Rabbi et al. 2011, Jaman et al. 2014, Jaman et al. 2015). But, these are not sufficient to visualize the overall status and condition of wildlife of nonprotected areas. The previous studies suggested that wildlife outside protected areas is facing numerous threats for human activities (e.g. illegal hunting, poaching, human wildlife conflict, etc.) (Jaman et 
al.2021, Islam et al. 2018, Khan 2015). Besides, many wild species are killed by human due to misconception and preconception in rural areas (Jaman et al. 2020). A baseline survey is essential for getting proper ideas about vertebrate wildlife in an area and implementing proper management plan for conserving them. The recent record of new species along with new geographical records indicates the importance of proper management system in non-protected areas (Hakim et al. 2020, Shome et al. 2020a, Kajol et al. 2020).

To get an overview of the wildlife diversity, we conducted a vertebrate wildlife survey in Sreepur upazila, Magura. No previous research study has been done on wildlife in this area. This study will provide a baseline information on wildlife status, abundance, seasonal occurrence, habitat usage and threats to vertebrate wildlife in Sreepur upazila, Magura, Bangladesh.

\section{Study area}

\section{MATERIAL AND METHODS}

The study area is $179.18 \mathrm{Km}^{2}$ under the Magura district of Khulna division (Fig. 1). It is located about $176 \mathrm{~km}$ south-west from Dhaka city. It is located in between $23^{\circ} 32^{\prime}$ and $23^{\circ} 41^{\prime}$ north latitudes and in between $89^{\circ} 21^{\prime}$ and $89^{\circ} 32^{\prime}$ east longitudes. The study area is mainly occupied by the homestead forests and crops lands.

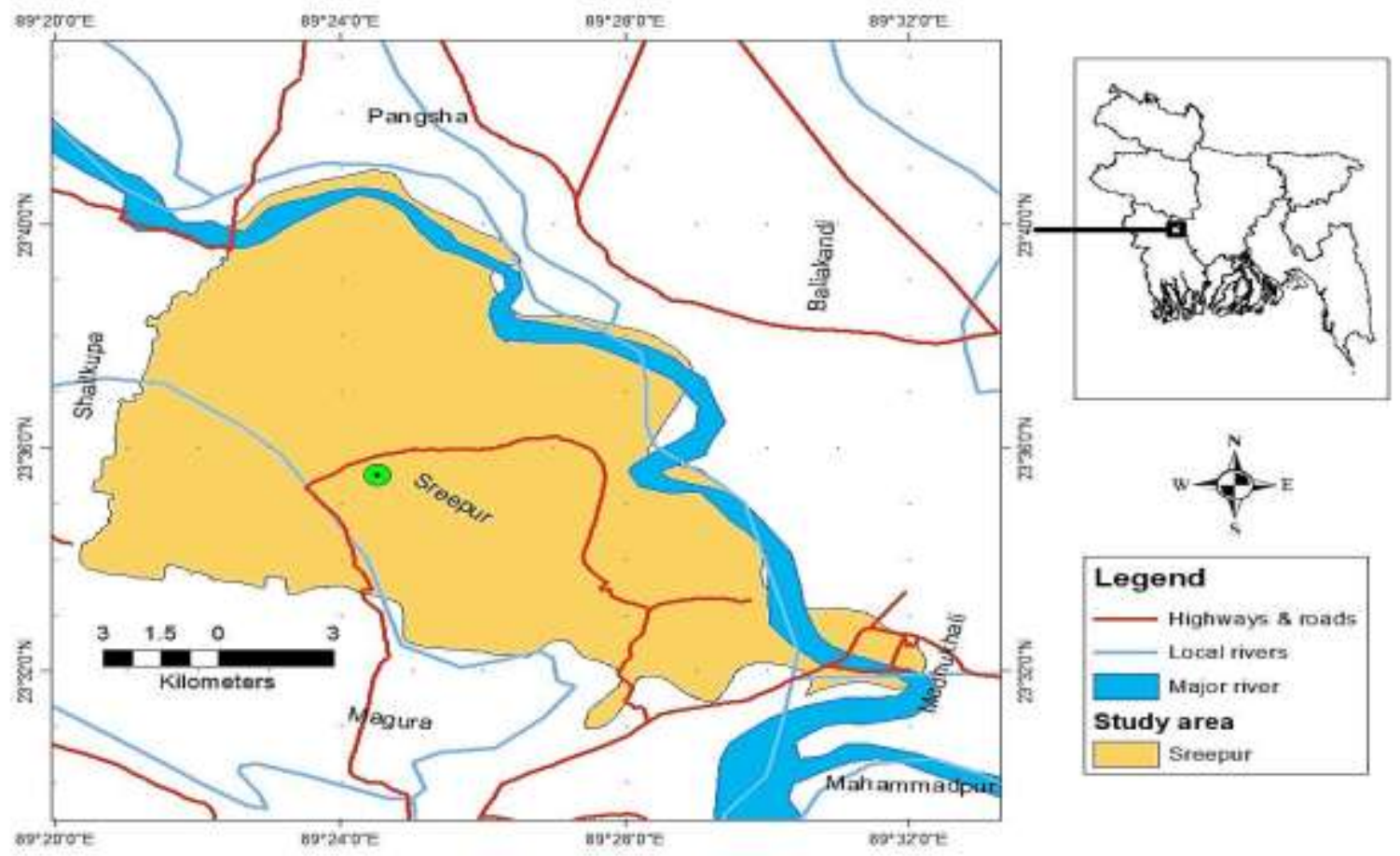

Fig. 1. Map of Sreepur upazila, Magura district -showing the study area.

\section{Observation techniques}

The study was based on direct field observation. Data collection was started in May 2015 and continued to April 2016. The study period was divided into three seasons, such as summer (MarchJune), rainy (July-October) and winter (November-February). The observation was made for four days in a month and each observation day was divided into three quarters. Sometimes night survey was conducted to observe nocturnal species. The observation usually started early in the morning and followed by afternoon, spent about 8 hours in a day. 


\section{Transect line method}

The study area was visited in north-south direction and in total 70 transect lines were made to observe the wildlife and their habitat utilizations. The size of each transect was one $\mathrm{km}$ in length and $200 \mathrm{~m}$ wide at both sides. The same speed and direction were repeated every time.

\section{Plot counting method}

For observing the amphibian and reptilian fauna, plot counting method was used. The counting of these animals was managed in various ways, depending on the species and habitat types in the plots. A total of 50 random plots was selected in the study area. Each plot size was $200 \mathrm{~m} \times 200 \mathrm{~m}$.

\section{Interview of local people}

All animals are not visible in all the year round, especially nocturnal animals are not easy to observe. So, interview of the local people was taken during the study period to know about those species that are present in the study area, but not easy to observe. Sometimes the calls and songs were recorded and identified by the respective experts. The activities of different species were observed directly or with the help of a pair of binoculars. Camera with different lens was used to photograph the habitats utilized by different vertebrate wildlife. Different field guides were used to identify wildlife in the fields (Khan 2018, Halder 2010, Khan 2015).

The relative abundance of a particular species was calculated by dividing the number of individuals of that species by total number of individuals of all species multiplied by 100 . To find out density of a species Khan (2015) was followed. We plotted rank-abundance curves for each group of wildlife by plotting the overall abundance against their rank following Whittaker (1965).

\section{Species richness, abundance and density}

\section{RESULTS AND DISCUSSION}

A total of 123 species of vertebrate wildlife was recorded from the study area during the study period. Among the total observed wildlife, amphibians were $6.50 \%$ (8 species), reptilians $10.57 \%$ (13 species), birds $68.29 \%$ (84 species) and mammals were $14.64 \%$ (18 species) (Table 1). Sarker et al. (2009) recorded 27 species of birds from two urban sites of Uttara, Dhaka. Rahman et al. (2013) reported 89 species of wild animals of which, six (6.74\%) species were amphibians, $11(12.36 \%)$ reptiles, 56 (62.93\%) birds and 16 (17.94\%) mammals from Sirajgonj, Bangladesh. Shome et al. (2020a) reported in total 140 species of birds belonging to 18 orders and 48 families were observed from Magura Sadar upazila. Another study recorded 209 species belonging to 79 families of wildlife from Dhaka urban areas, of them 12 species were amphibians, 19 reptiles, 162 birds and 16 mammals (Jaman et al. 2021). In total 4352 individuals of wildlife were counted from the study area. Among them, 1.79\% $(\mathrm{n}=78)$ were amphibians, $1.90 \%(\mathrm{n}=83)$ reptilians, $89.65 \% \quad(\mathrm{n}=3902)$ birds and 6.64\% $(\mathrm{n}=289)$ mammals.

\section{Amphibians}

All recorded amphibian species were frogs and toad belonging to five families (Table 1). The highest abundance $(\mathrm{n}=20)$ and density $\left(10 / \mathrm{km}^{2}\right)$ was recorded for Indian bull frog (Hoplobatrachus tigerinus). The least abundance $(\mathrm{n}=5)$ and density $\left(2.5 / \mathrm{km}^{2}\right)$ was recorded for maculated tree frog (Polypedates maculates) and (Microhyla sp.). Indian bull frog is distributed widely in Bangladesh and can occur in every type of habitats including urban landscapes (Hasan et al. 2014). Thus, we also observed high number of individual of this species during the study. 


\section{Reptiles}

Among the observed reptilian species, six (46.15\%) species were snakes and seven (53.85\%) were lizards (Table 1). No turtle species was observed or recorded through the questionnaire survey. The top most abundance $(\mathrm{n}=26)$ and density $\left(13 / \mathrm{km}^{2}\right)$ was calculated for house lizard (Hemidactylus flaviviridis) whereas the lowest abundance $(\mathrm{n}=1)$ and density $\left(0.5 / \mathrm{km}^{2}\right)$ was found for Indian rat snake (Ptyas muсosa). House lizard is an adaptive gecko species that can occur in and around human habitations as well as in forest habitats (Jaman et al. 2021). This kind of adaptation caused this reptile species to be the most abundant species in the area.

\section{Birds}

Diverse group of birds in term of feeding behaviour was observed. The number of the Passerine bird species was $45(53.57 \%)$ and of the non-passerines 39 (46.43\%). Among the recorded bird species, 72 $(85.72 \%)$ species were resident and $12(14.28 \%)$ were migratory. The maximum abundance $(\mathrm{n}=752)$ and density $\left(26.85 / \mathrm{km}^{2}\right)$ was recorded for common myna (Acridotheres tristis) and the minimum abundance $(\mathrm{n}=1)$ and density $\left(0.036 / \mathrm{km}^{2}\right)$ was for orange-headed thrush (Zoothera citrina) (Table 1). Common myna is an omnivorous, highly adaptive and scavenger bird. This species can tolerate habitat alteration and found throughout the year (Khan 2015). During sampling this species was found in the study area frequently in different microhabitats. Shome et al. (2020) recorded 140 species of birds from Magura Sadar upazila, a small site where bird diversity was higher than the birds recorded in this study.

\section{Mammals}

Among the mammals, the highest observed population $(n=91)$ was for large bandicoot rat (Bandicota indica) and the density was $3.25 / \mathrm{km}^{2}$. The lower most abundance $(\mathrm{n}=2)$ and density $\left(0.07 / \mathrm{km}^{2}\right)$ was for fishing cat (Prionailurus viverrinus). Most of the observed mammals were rodents and their total individuals were also higher compared to the carnivorous and flying mammals (Table 1). This indicates that the study area is a suitable ground for the vertebrate pest animals like rats and mice.

Table 1. Recorded vertebrate wildlife in Sreepur upazila, Magura with the status of each species.

\begin{tabular}{|c|c|c|c|c|c|c|c|c|c|c|c|}
\hline $\begin{array}{l}\text { Scientific } \\
\text { name }\end{array}$ & $\begin{array}{l}\text { Common } \\
\text { name }\end{array}$ & $\mathbf{N}$ & RA & D & $\mathbf{S}$ & Scientific name & Common name & $\mathbf{N}$ & RA & D & OS \\
\hline \multicolumn{12}{|c|}{ Amphibia (8 species) } \\
\hline $\begin{array}{l}\text { Hoplobatrachus } \\
\text { tigerinus }\end{array}$ & $\begin{array}{l}\text { Indian bull } \\
\text { frog }\end{array}$ & 20 & 25.64 & 10 & $\mathrm{C}$ & $\begin{array}{l}\text { Microhyla } \\
\text { ornata }\end{array}$ & $\begin{array}{l}\text { Ornate narrow- } \\
\text { mouthed frog }\end{array}$ & 8 & 10.26 & 4 & $\mathrm{~F}$ \\
\hline $\begin{array}{l}\text { Euphlyctis } \\
\text { cyanophlyctis }\end{array}$ & Skipper frog & 14 & 17.95 & 7 & $\mathrm{C}$ & $\begin{array}{l}\text { Polypedates } \\
\text { leucomystax }\end{array}$ & $\begin{array}{l}\text { Common tree } \\
\text { frog }\end{array}$ & 7 & 8.97 & 3.5 & $\mathrm{~F}$ \\
\hline $\begin{array}{l}\text { Duttaphrynus } \\
\text { melanostictus }\end{array}$ & $\begin{array}{l}\text { Asian common } \\
\text { toad }\end{array}$ & 10 & 12.82 & 5 & FC & Microhyla sp. & $\begin{array}{l}\text { Narrow-mouthed } \\
\text { frog }\end{array}$ & 5 & 6.41 & 2.5 & $\mathrm{~F}$ \\
\hline $\begin{array}{l}\text { Fejervarya } \\
\text { asmati }\end{array}$ & $\begin{array}{l}\text { Asmat's } \\
\text { cricket frog }\end{array}$ & 9 & 11.54 & 4.5 & FC & $\begin{array}{c}\text { Polypdates } \\
\text { maculatus }\end{array}$ & $\begin{array}{l}\text { Maculated } \\
\text { tree frog }\end{array}$ & 5 & 6.41 & 2.5 & $\mathrm{~F}$ \\
\hline \multicolumn{12}{|c|}{ Reptilia (13 species) } \\
\hline $\begin{array}{l}\text { Hemidactylus } \\
\text { flaviviridis }\end{array}$ & House lizard & 26 & 31.33 & 13 & $\mathrm{C}$ & $\begin{array}{l}\text { *Amphiesma } \\
\text { stolatum }\end{array}$ & Striped keelback & 3 & 3.61 & 1.5 & $\mathrm{~F}$ \\
\hline $\begin{array}{l}\text { Xenochrophis } \\
\text { piscator }\end{array}$ & $\begin{array}{l}\text { Checkered } \\
\text { keelback }\end{array}$ & 13 & 15.66 & 6.5 & FC & *Gekko gecko & Tokay gecko & 3 & 3.61 & 1.5 & $\mathrm{~F}$ \\
\hline $\begin{array}{l}\text { Calotes } \\
\text { versicolor }\end{array}$ & $\begin{array}{l}\text { Common } \\
\text { garden lizard }\end{array}$ & 12 & 14.46 & 6 & FC & *Naja naja & Binocellate cobra & 2 & 2.41 & 1 & $\mathrm{~F}$ \\
\hline $\begin{array}{l}\text { Eutropis } \\
\text { carinata }\end{array}$ & $\begin{array}{l}\text { Common } \\
\text { skink }\end{array}$ & 6 & 7.23 & 3 & $\mathrm{~F}$ & $\begin{array}{l}\text { *Ahaetulla } \\
\text { nasuta }\end{array}$ & Vine snake & 2 & 2.41 & 1 & $\mathrm{~F}$ \\
\hline Varanus & Yellow & 5 & 6.02 & 2.5 & $\mathrm{~F}$ & *Typhlops & Diard's & 2 & 2.41 & 1 & $\mathrm{~F}$ \\
\hline
\end{tabular}




\begin{tabular}{|c|c|c|c|c|c|c|c|c|c|c|c|}
\hline flavescens & monitor & & & & & diardii & Blind snake & & & & \\
\hline $\begin{array}{l}\text { Hemidactylus } \\
\text { brookii }\end{array}$ & $\begin{array}{l}\text { Brook's } \\
\text { house gecko }\end{array}$ & 5 & 6.02 & 2.5 & $\mathrm{~F}$ & *Ptyas mисоsa & Indian rat snake & 1 & 1.2 & 0.5 & $\mathrm{~F}$ \\
\hline $\begin{array}{l}\text { *Varanus } \\
\text { bengalensis }\end{array}$ & $\begin{array}{l}\text { Bengal } \\
\text { monitor }\end{array}$ & 3 & 3.61 & 1.5 & $\mathrm{~F}$ & & & & & & \\
\hline \multicolumn{12}{|c|}{ Aves (84 species) } \\
\hline $\begin{array}{l}\text { Acridotheres } \\
\text { tristis }\end{array}$ & $\begin{array}{l}\text { Common } \\
\text { myna }\end{array}$ & 752 & 19.27 & 26.85 & $\mathrm{VC}$ & Lonchura striata & $\begin{array}{l}\text { White-rumped } \\
\text { munia }\end{array}$ & 10 & 0.26 & 0.36 & $\mathrm{~F}$ \\
\hline $\begin{array}{l}\text { Pycnonotus } \\
\text { cafer }\end{array}$ & $\begin{array}{l}\text { Red-vented } \\
\text { bulbul }\end{array}$ & 628 & 16.09 & 22.43 & $\mathrm{VC}$ & $\begin{array}{l}\text { Coracias } \\
\text { benghalensis }\end{array}$ & Indian roller & 9 & 0.23 & 0.32 & $\bar{F}$ \\
\hline $\begin{array}{l}\text { Dicrurus } \\
\text { macrocercus }\end{array}$ & Black drongo & 326 & 8.35 & 11.64 & $\mathrm{C}$ & $\begin{array}{l}\text { Lonchura } \\
\text { malabarica }\end{array}$ & Indian silverbill & 9 & 0.23 & 0.32 & $\mathrm{~F}$ \\
\hline $\begin{array}{l}\text { Acridotheres } \\
\text { fuscus }\end{array}$ & Jungle myna & 281 & 7.2 & 10.03 & $\mathrm{C}$ & Milvus migrans & Black kite & 9 & 0.23 & 0.32 & $\mathrm{~F}$ \\
\hline Apus affinis & House swift & 205 & 5.25 & 7.32 & $\mathrm{C}$ & Ardea alba & Great white wgret & 9 & 0.23 & 0.32 & $\mathrm{~F}$ \\
\hline Sturnus contra & Pied myna & 173 & 4.43 & 6.18 & $\mathrm{C}$ & $\begin{array}{l}\text { Hierococcyx } \\
\text { varius }\end{array}$ & $\begin{array}{l}\text { Common hawk- } \\
\text { cuckoo }\end{array}$ & 8 & 0.21 & 0.28 & $\mathrm{~F}$ \\
\hline $\begin{array}{l}\text { Passer } \\
\text { domesticus }\end{array}$ & $\begin{array}{l}\text { House } \\
\text { sparrow }\end{array}$ & 141 & 3.61 & 5.03 & FC & $\begin{array}{l}\text { Cacomantis } \\
\text { merulinus }\end{array}$ & Plaintive cuckoo & 8 & 0.21 & 0.28 & $\bar{F}$ \\
\hline Coracina macei & $\begin{array}{l}\text { Large cuckoo } \\
\text { shrike }\end{array}$ & 97 & 2.49 & 3.46 & $\mathrm{FC}$ & $\begin{array}{l}\text { Cypsiurus } \\
\text { balasiensis }\end{array}$ & Asian palm swift & 7 & 0.18 & 0.25 & $\bar{F}$ \\
\hline $\begin{array}{l}\text { Centropus } \\
\text { sinensis }\end{array}$ & $\begin{array}{l}\text { Greater } \\
\text { coucal }\end{array}$ & 93 & 2.38 & 3.32 & FC & $\begin{array}{l}\text { Nectarinia } \\
\text { asiaticus }\end{array}$ & Purple sunbird & 7 & 0.18 & 0.25 & $\bar{F}$ \\
\hline $\begin{array}{l}\text { Cyornis } \\
\text { rubeculoides }\end{array}$ & $\begin{array}{l}\text { Blue-throated } \\
\text { blue flycatcher }\end{array}$ & 88 & 2.26 & 3.14 & FC & $\begin{array}{l}\text { Cyaneculas } \\
\text { vecica }\end{array}$ & Blue throat & 7 & 0.18 & 0.25 & $\mathrm{~F}$ \\
\hline $\begin{array}{l}\text { Copsychus } \\
\text { saularis }\end{array}$ & $\begin{array}{l}\text { Oriental } \\
\text { magpie-robin }\end{array}$ & 86 & 2.2 & 3.07 & FC & Athene brama & Spotted owlet & 7 & 0.18 & 0.25 & $\mathrm{~F}$ \\
\hline $\begin{array}{l}\text { Dinopium } \\
\text { benghalense }\end{array}$ & $\begin{array}{l}\text { Black-rumped } \\
\text { Flameback }\end{array}$ & 65 & 1.67 & 2.32 & FC & $\begin{array}{l}\text { Anastomus } \\
\text { oscitans }\end{array}$ & Asian Openbill & 7 & 0.18 & 0.25 & $\mathrm{~F}$ \\
\hline $\begin{array}{l}\text { Coracina } \\
\text { melanoptera }\end{array}$ & $\begin{array}{l}\text { Black-headed } \\
\text { cuckooshrike }\end{array}$ & 64 & 1.64 & 2.28 & $\mathrm{~F}$ & $\begin{array}{l}\text { Copsychus } \\
\text { malabaricus }\end{array}$ & $\begin{array}{l}\text { White-rumped } \\
\text { shama }\end{array}$ & 6 & 0.15 & 0.21 & $\mathrm{~F}$ \\
\hline $\begin{array}{c}\text { Spilopelia } \\
\text { chinensis }\end{array}$ & $\begin{array}{l}\text { Eastern } \\
\text { spotted dove }\end{array}$ & 55 & 1.41 & 1.96 & $\mathrm{~F}$ & $\begin{array}{l}\text { Dendrocopos } \\
\text { macei }\end{array}$ & $\begin{array}{l}\text { Fulvous-breasted } \\
\text { woodpecker }\end{array}$ & 6 & 0.15 & 0.21 & $\mathrm{~F}$ \\
\hline Ardeola grayii & Pond heron & 48 & 1.23 & 1.71 & FC & Haliastur indus & Brahmany kite & 6 & 0.15 & 0.21 & $\mathrm{~F}$ \\
\hline $\begin{array}{l}\text { Halcyon } \\
\text { smyrnensis }\end{array}$ & $\begin{array}{l}\text { White-breasted } \\
\text { kingfisher }\end{array}$ & 44 & 1.13 & 1.57 & $\mathrm{~F}$ & $\begin{array}{l}\text { Motacilla } \\
\text { madaraspatensis }\end{array}$ & $\begin{array}{l}\text { White-browed } \\
\text { wagtail }\end{array}$ & 6 & 0.15 & 0.21 & $\mathrm{~F}$ \\
\hline Alcedo atthis & $\begin{array}{l}\text { Common } \\
\text { kingfisher }\end{array}$ & 42 & 1.08 & 1.5 & FC & Parus major & Great tit & 6 & 0.15 & 0.21 & $\bar{F}$ \\
\hline Aegithina tiphia & Common iora & 33 & 0.85 & 1.17 & FC & $\begin{array}{l}\text { Megalaima } \\
\text { haemacephala }\end{array}$ & $\begin{array}{l}\text { Coppersmith } \\
\text { barbet }\end{array}$ & 5 & 0.13 & 0.18 & $\mathrm{~F}$ \\
\hline $\begin{array}{l}\text { Dicaeum } \\
\text { erythrorhynchos }\end{array}$ & $\begin{array}{l}\text { Pale-bellied } \\
\text { flowerpecker }\end{array}$ & 33 & 0.85 & 1.17 & FC & Prinia inornata & Plain prinia & 5 & 0.13 & 0.18 & $\mathrm{~F}$ \\
\hline $\begin{array}{l}\text { Micropternus } \\
\text { brachyurus }\end{array}$ & $\begin{array}{l}\text { Rufous } \\
\text { woodpecker }\end{array}$ & 32 & 0.82 & 1.14 & FC & $\begin{array}{l}\text { Nectarinia } \\
\text { zeylonica }\end{array}$ & $\begin{array}{l}\text { Purple-rumped } \\
\text { sunbird }\end{array}$ & 5 & 0.13 & 0.18 & $\mathrm{~F}$ \\
\hline Bubulcus ibis & Cattle egret & 32 & 0.82 & 1.14 & $\mathrm{~F}$ & $\begin{array}{l}\text { Streptopelia } \\
\text { tranquebarica }\end{array}$ & Red turtle dove & 5 & 0.13 & 0.18 & $\mathrm{~F}$ \\
\hline $\begin{array}{l}\text { Phylloscopus } \\
\text { affinis }\end{array}$ & $\begin{array}{l}\text { Tickell's } \\
\text { leaf-warbler }\end{array}$ & 30 & 0.77 & 1.07 & FC & Tyto alba & Barn owl & 5 & 0.13 & 0.17 & $\mathrm{~F}$ \\
\hline $\begin{array}{l}\text { Turdoides } \\
\text { striata }\end{array}$ & $\begin{array}{l}\text { Jungle } \\
\text { babbler }\end{array}$ & 28 & 0.72 & 1 & FC & $\begin{array}{l}\text { Acrocephalus } \\
\text { dumetorum }\end{array}$ & $\begin{array}{l}\text { Blyth's reed- } \\
\text { warbler }\end{array}$ & 5 & 0.13 & 0.17 & $\mathrm{~F}$ \\
\hline $\begin{array}{l}\text { Oriolus } \\
\text { xanthornus }\end{array}$ & $\begin{array}{l}\text { Black-hooded } \\
\text { oriole }\end{array}$ & 28 & 0.72 & 1 & $\mathrm{~F}$ & $\begin{array}{l}\text { Corvus } \\
\text { splendens }\end{array}$ & House crow & 4 & 0.1 & 0.14 & $\mathrm{~F}$ \\
\hline $\begin{array}{l}\text { Sturnus } \\
\text { malabaricus }\end{array}$ & $\begin{array}{l}\text { Chestnut-tailed } \\
\text { starling }\end{array}$ & 27 & 0.69 & 0.96 & $\mathrm{~F}$ & $\begin{array}{l}\text { Pericrocotus } \\
\text { cinnamomeus }\end{array}$ & Small minivet & 4 & 0.1 & 0.14 & $\bar{F}$ \\
\hline
\end{tabular}




\begin{tabular}{|c|c|c|c|c|c|c|c|c|c|c|c|}
\hline $\begin{array}{l}\text { Megalaima } \\
\text { asiatica }\end{array}$ & $\begin{array}{l}\text { Blue-throated } \\
\text { barbet }\end{array}$ & 26 & 0.67 & 0.93 & $\mathrm{~F}$ & $\begin{array}{l}\text { Psittacula } \\
\text { cyanocephala }\end{array}$ & $\begin{array}{l}\text { Plum-headed } \\
\text { parakeet }\end{array}$ & 4 & 0.1 & 0.14 & $\mathrm{~F}$ \\
\hline $\begin{array}{l}\text { Dendrocitta } \\
\text { vagabunda }\end{array}$ & $\begin{array}{l}\text { Rufous } \\
\text { treepie }\end{array}$ & 22 & 0.56 & 0.78 & $\mathrm{~F}$ & Motacilla alba & White wagtail & 4 & 0.1 & 0.14 & $\mathrm{~F}$ \\
\hline $\begin{array}{l}\text { Psilopogon } \\
\text { lineata }\end{array}$ & $\begin{array}{l}\text { Lineated } \\
\text { barbet }\end{array}$ & 20 & 0.51 & 0.71 & $\mathrm{~F}$ & $\begin{array}{l}\text { Picus } \\
\text { xanthopygaeus }\end{array}$ & $\begin{array}{l}\text { Streak-throated } \\
\text { woodpecker }\end{array}$ & 4 & 0.1 & 0.14 & $\mathrm{~F}$ \\
\hline $\begin{array}{l}\text { Ardea } \\
\text { intermedia }\end{array}$ & $\begin{array}{l}\text { Intermediate } \\
\text { egret }\end{array}$ & 19 & 0.49 & 0.68 & $\mathrm{~F}$ & $\begin{array}{l}\text { Ploceus } \\
\text { phillippinus }\end{array}$ & Baya weaver & 4 & 0.1 & 0.14 & $\mathrm{~F}$ \\
\hline $\begin{array}{l}\text { Pellorneum } \\
\text { ruficeps }\end{array}$ & $\begin{array}{l}\text { Puff-throated } \\
\text { babbler }\end{array}$ & 19 & 0.49 & 0.68 & $\mathrm{~F}$ & $\begin{array}{l}\text { Corvus } \\
\text { levaillantii }\end{array}$ & Jungle crow & 3 & 0.08 & 0.11 & $\mathrm{~F}$ \\
\hline $\begin{array}{l}\text { Orthotomus } \\
\text { sutorius }\end{array}$ & $\begin{array}{l}\text { Common } \\
\text { tailorbird }\end{array}$ & 17 & 0.44 & 0.61 & $\mathrm{~F}$ & $\begin{array}{l}\text { *Dicrurus } \\
\text { aeneus }\end{array}$ & Bronzed drongo & 3 & 0.08 & 0.11 & $\mathrm{~F}$ \\
\hline Otus lettia & $\begin{array}{l}\text { Collared } \\
\text { scops owl }\end{array}$ & 17 & 0.44 & 0.61 & $\mathrm{~F}$ & $\begin{array}{l}\text { *Psittacula } \\
\text { krameri }\end{array}$ & $\begin{array}{l}\text { Rose-ringed } \\
\text { parakeet }\end{array}$ & 3 & 0.08 & 0.11 & $\mathrm{~F}$ \\
\hline $\begin{array}{l}\text { Lonchura } \\
\text { punctulata }\end{array}$ & $\begin{array}{l}\text { Scaly-breasted } \\
\text { munia }\end{array}$ & 15 & 0.38 & 0.53 & $\mathrm{~F}$ & $\begin{array}{l}* \text { Saxicola } \\
\text { caprata }\end{array}$ & Pied bushchat & 3 & 0.08 & 0.11 & $\mathrm{~F}$ \\
\hline $\begin{array}{l}\text { Merops } \\
\text { orientalis }\end{array}$ & $\begin{array}{l}\text { Asian Green } \\
\text { bee-eater }\end{array}$ & 13 & 0.33 & 0.46 & $\mathrm{~F}$ & $*$ Oriolus oriolus & $\begin{array}{l}\text { Eurasian golden } \\
\text { oriole }\end{array}$ & 3 & 0.08 & 0.11 & $\mathrm{~F}$ \\
\hline Egretta garzetta & Little egret & 12 & 0.31 & 0.43 & $\mathrm{~F}$ & *Jynx torquilla & Eurasian wryneck & 3 & 0.08 & 0.11 & $\mathrm{~F}$ \\
\hline $\begin{array}{l}\text { Eudynamys } \\
\text { scolopaceus }\end{array}$ & Western koel & 12 & 0.31 & 0.43 & $\mathrm{~F}$ & $\begin{array}{l}\text { *Phalacrocorax } \\
\text { niger }\end{array}$ & Little cormorant & 3 & 0.08 & 0.11 & $\mathrm{~F}$ \\
\hline $\begin{array}{l}\text { Clamator } \\
\text { jacobinus }\end{array}$ & $\begin{array}{l}\text { Jacobin } \\
\text { cuckoo }\end{array}$ & 12 & 0.31 & 0.43 & $\mathrm{~F}$ & $\begin{array}{l}{ }^{* \text { Centropus }} \\
\text { bengalensis }\end{array}$ & Lesser coucal & 3 & 0.08 & 0.11 & $\mathrm{~F}$ \\
\hline $\begin{array}{l}\text { Picoides } \\
\text { canicapillus }\end{array}$ & $\begin{array}{l}\text { Grey-capped } \\
\text { woodpecker }\end{array}$ & 11 & 0.28 & 0.39 & $\mathrm{~F}$ & $\begin{array}{l}\text { *Tephrodornis } \\
\text { pondicerianus }\end{array}$ & $\begin{array}{l}\text { Common } \\
\text { woodshrike }\end{array}$ & 2 & 0.05 & 0.07 & $\mathrm{~F}$ \\
\hline $\begin{array}{l}\text { Gecinulus } \\
\text { grantia }\end{array}$ & $\begin{array}{l}\text { Pale-headed } \\
\text { woodpecker }\end{array}$ & 11 & 0.28 & 0.39 & $\mathrm{~F}$ & $\begin{array}{l}\text { *Zosterops } \\
\text { palpebrosus }\end{array}$ & $\begin{array}{l}\text { Oriental white- } \\
\text { eye }\end{array}$ & 2 & 0.05 & 0.07 & $\mathrm{~F}$ \\
\hline Picus guerini & $\begin{array}{l}\text { Black-naped } \\
\text { woodpecker }\end{array}$ & 11 & 0.28 & 0.39 & $\mathrm{~F}$ & $\begin{array}{l}\text { *Acrocephalus } \\
\text { agricola }\end{array}$ & $\begin{array}{l}\text { Paddyfield } \\
\text { warbler }\end{array}$ & 2 & 0.05 & 0.07 & $\mathrm{~F}$ \\
\hline $\begin{array}{l}\text { Ficedula } \\
\text { albicilla }\end{array}$ & $\begin{array}{l}\text { Taiga } \\
\text { flycatcher }\end{array}$ & 10 & 0.26 & 0.36 & $\mathrm{~F}$ & $\begin{array}{l}\text { *Merops } \\
\text { philippinus }\end{array}$ & $\begin{array}{l}\text { Blue-tailed bee- } \\
\text { eater }\end{array}$ & 2 & 0.05 & 0.07 & $\mathrm{~F}$ \\
\hline Lanius cristatus & Brown shrike & 10 & 0.26 & 0.36 & $\mathrm{~F}$ & $\begin{array}{l}\text { *Zoothera } \\
\text { citrina }\end{array}$ & $\begin{array}{l}\text { Orange-headed } \\
\text { trush }\end{array}$ & 1 & 0.03 & 0.03 & $\mathrm{~F}$ \\
\hline \multicolumn{12}{|c|}{ Mammalia (18 species) } \\
\hline $\begin{array}{l}\text { Bandicota } \\
\text { indica }\end{array}$ & $\begin{array}{l}\text { Large } \\
\text { bandicoot rat }\end{array}$ & 91 & 31.49 & 3.25 & $\mathrm{VC}$ & Mus booduga & $\begin{array}{l}\text { Little Indian } \\
\text { field mouse }\end{array}$ & 7 & 2.42 & 0.25 & FC \\
\hline $\begin{array}{l}\text { Funambulus } \\
\text { pennantii }\end{array}$ & $\begin{array}{l}\text { Northern } \\
\text { palm squirrel }\end{array}$ & 72 & 24.91 & 2.57 & $\mathrm{C}$ & $\begin{array}{l}\text { Pipistrellus } \\
\text { coromandra }\end{array}$ & Indian pipistrelle & 6 & 2.08 & 0.21 & FC \\
\hline $\begin{array}{l}\text { Bandicota } \\
\text { bengalensis }\end{array}$ & $\begin{array}{l}\text { Lesser } \\
\text { bandicoot rat }\end{array}$ & 16 & 5.54 & 0.57 & $\mathrm{FC}$ & $\begin{array}{l}\text { Rattus } \\
\text { norvegicus }\end{array}$ & Brown rat & 5 & 1.73 & 0.18 & FC \\
\hline $\begin{array}{l}\text { Herpestes } \\
\text { edwardsii }\end{array}$ & $\begin{array}{l}\text { Common } \\
\text { mongoose }\end{array}$ & 16 & 5.54 & FC & $\mathrm{FC}$ & $\begin{array}{l}\text { Pteropus } \\
\text { giganteus }\end{array}$ & Indian flying fox & 4 & 1.38 & 0.14 & $\mathrm{~F}$ \\
\hline Suncus murinus & $\begin{array}{l}\text { Asian house } \\
\text { shrew }\end{array}$ & 14 & 4.84 & 0.5 & $\mathrm{C}$ & $\begin{array}{l}\text { Pipistrellus } \\
\text { tenuis }\end{array}$ & Least pipistrelle & 4 & 1.38 & 0.14 & $\mathrm{~F}$ \\
\hline Rattus rattus & $\begin{array}{l}\text { Common } \\
\text { house rat }\end{array}$ & 13 & 4.5 & 0.46 & $\mathrm{FC}$ & *Felis chaus & Jungle cat & 4 & 1.38 & 0.14 & $\mathrm{~F}$ \\
\hline Mus musculus & House mouse & 10 & 3.46 & 0.36 & $\mathrm{FC}$ & $\begin{array}{l}\text { *Vulpes } \\
\text { bengalensis }\end{array}$ & Bengal fox & 4 & 1.38 & 0.14 & $\mathrm{~F}$ \\
\hline $\begin{array}{l}\text { Herpestes } \\
\text { auropunctatus }\end{array}$ & $\begin{array}{l}\text { Small Indian } \\
\text { mongoose }\end{array}$ & 10 & 3.46 & 0.57 & FC & *Canis aureus & Asiatic jackel & 3 & 1.04 & 0.11 & $\mathrm{~F}$ \\
\hline $\begin{array}{l}\text { Megaderma } \\
\text { lyra }\end{array}$ & $\begin{array}{l}\text { Greater false } \\
\text { vampire }\end{array}$ & 8 & 2.77 & 0.28 & $\mathrm{FC}$ & $\begin{array}{l}\text { *Prionailurus } \\
\text { viverrinus }\end{array}$ & Fishing cat & 2 & 0.69 & 0.07 & $\mathrm{~F}$ \\
\hline
\end{tabular}

[Note: N - Number of individual; RA - Relative Abundance; D - Density/ $\mathrm{km}^{2}$; S - Status, VC - Very Common, C- Common,

FC - Fairly Common; Few - F, LC- Least Concerned, NT- Near Threatened and EN- Endangered. Rare species observed in the study area is marked $(*)$ in the table.] 
Diverse species composition under various vertebrate wildlife groups indicates that this area consists by ideal habitats for wildlife. Some natural patchy habitats in the study area are suitable for providing food, shelter and breeding facilities of some particular type of wildlife. There is no previous research about wildlife of this study area. Some of the rare bird species is presented in Fig. 2.

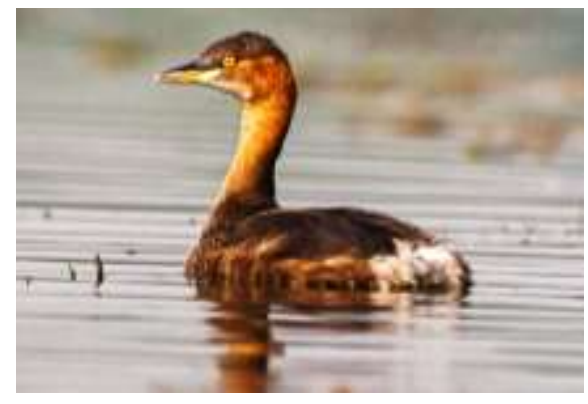

a.

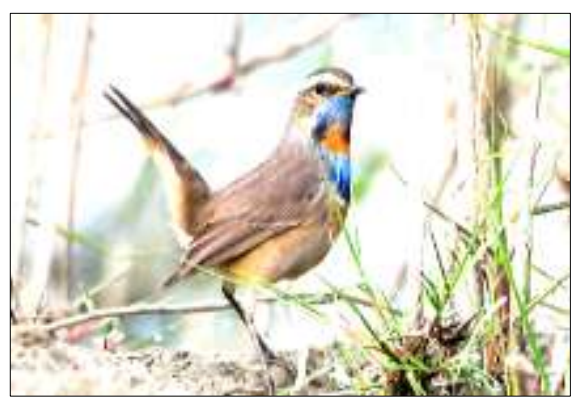

b.

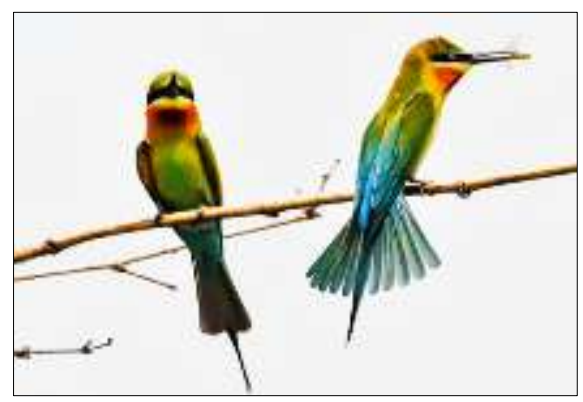

c.

Fig. 2. Some rare bird species observed in the site: a. Little Grebe (Tachbaptus ruficollis); b. Blue throat (Luscinia servica); and c. Blue-tailed bee-eater (Merops philippinus).

\section{Species richness in months and seasons}

The maximum species richness was recorded in December (62 species). The lowest species richness was recorded in June (33 species) (Fig. 3a). We found that there was a remarkable variation in the richness of species in seasons. Winter season (108 species) was the pick for species diversity of vertebrate wildlife in terms of richness followed by rainy (94 species) and summer (70 species) (Fig. $3 b)$. In winter, 10 species of migratory birds were observed in the study area. Migratory birds in winter may influence the richness and diversity of wildlife including birds in a region (Shome et al. 2020b). Additionally, food availability increased in agricultural lands, farmlands and water body during winter those attract wildlife to forage on them.

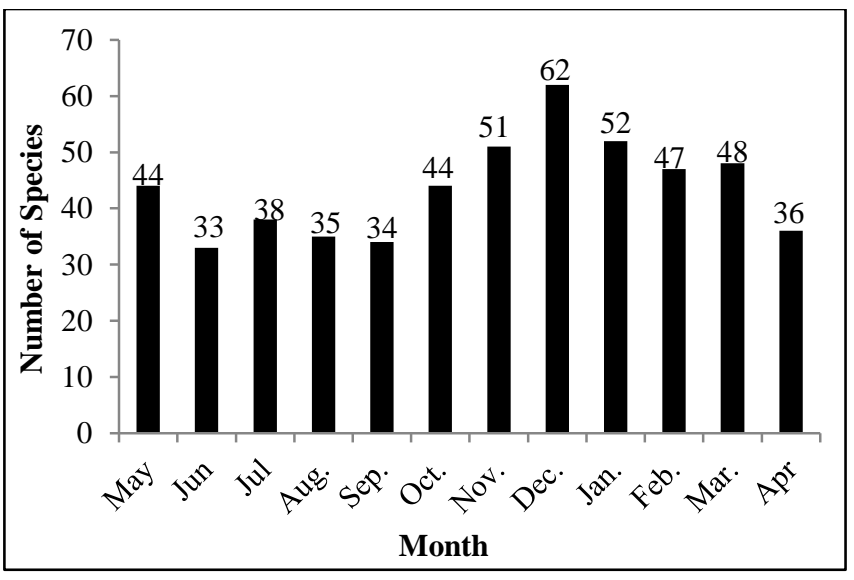

a.

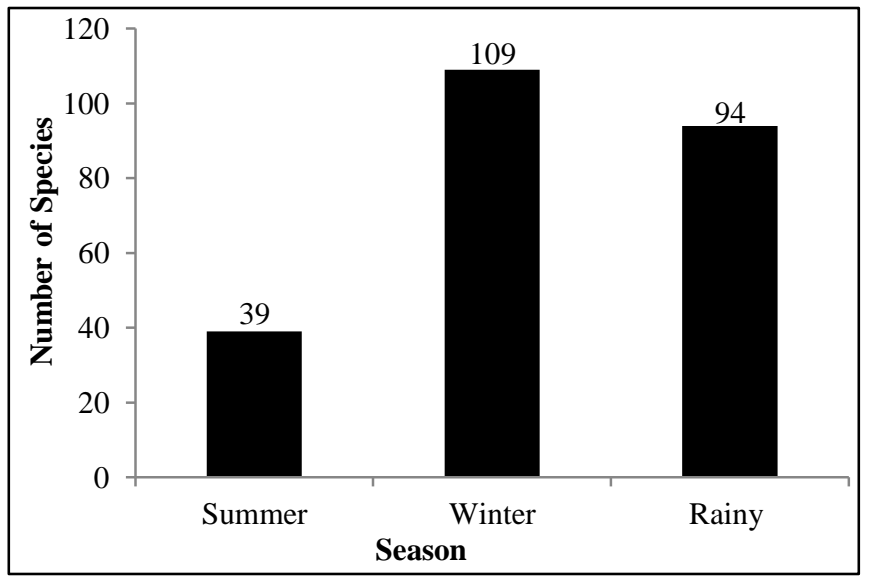

b.

Fig. 3. Showing species richness in study area: a. Species in months; and b. Species richness in seasons.

\section{Species richness across habitats}

Considering macro-habitats, we found that 76 species $(61.78 \%)$ preferred arboreal habitat such as trees followed by 31 species $(25.21 \%)$ in terrestrial habitat and 16 species $(13.01 \%)$ in aquatic habitat. Among 12 types of micro-habitats, tree was the most used microhabitat (68 species, 55\%) (Fig. 4). The study area was mostly occupied by various types of native plant species which might provide good 
shelter, food, roosting and nesting facilities for the wildlife. Besides, the species composition includes 84 bird species which is more than two-third of the total wildlife. Birds use tree for versatile purposes, thus can manipulate the result of microhabitat usage in this study.

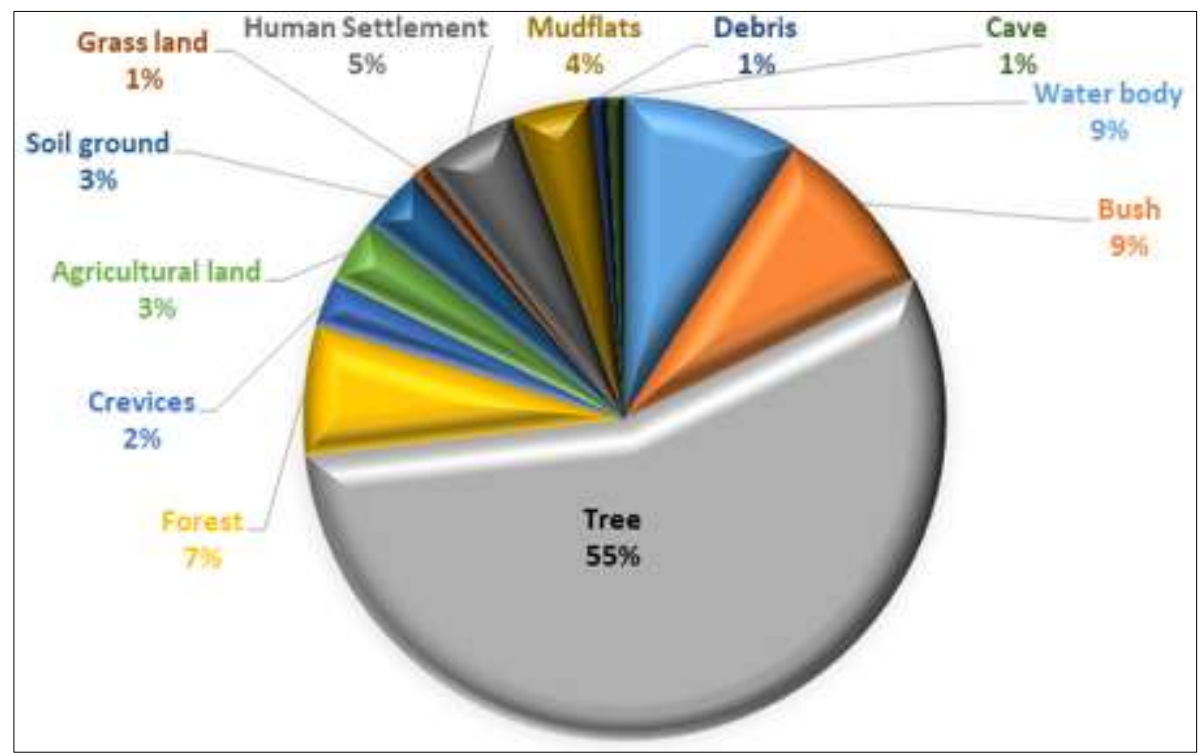

Fig. 4. Species richness in the different microhabitats.

Observation status, relative abundance, rank-abundance curve, and threatened status

Among the recorded wildlife, the observation status showed that three species $(2.44 \%)$ were very common, nine (7.32\%) were common, 26 (21.14\%) were fairly common and $85(69.1 \%)$ were few (Table 1).

\section{Amphibians}

Among all amphibians, the relative abundance of Hoplobatrachus tigerinus was the highest (25.64\%) and it also ranked topmost amphibians in the curve (Table 1 and Fig. 5a). The study area holds many water resources that act as foraging and breeding ground like other areas for this species (Hasan $e t$ al. 2014.

\section{Reptiles}

The relative abundance was the highest for common house gecko Hemidactylus flaviviridis (31.33\%) and it is also placed at the apex position in the curve followed by checkered keelback Xenochrophis piscator (15.66\%) (Table 1 and Fig. 5b). It was observed that this study site is suitable for Hemidactylus flaviviridis as there are many old buildings, household and forested habitats. Alongside, water bodies like lakes, ponds, beel, canals, ditches provide a good support for Xenochrophis piscator.

\section{Birds}

The relative abundance of Acridotheres tristis was the highest (19.27\%) followed by Pycnonotus cafer $(16.09 \%)$ as they are supported by suitable native and planted tree species, homestead gardens, agricultural lands and soil ground providing enough food, shelter and nesting facilities for them. Acridotheres tristis also ranked as the most dominant bird species in rank-abundance curve (Fig. 5c). 


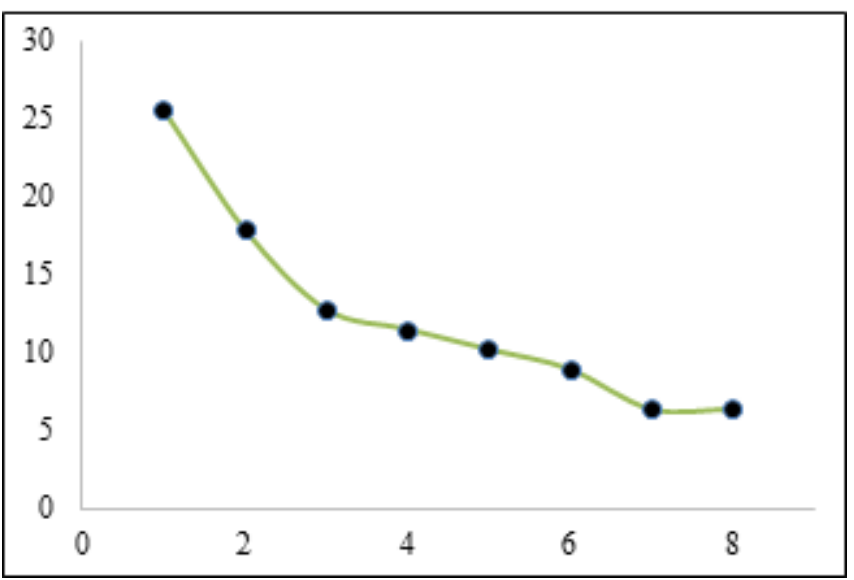

a.

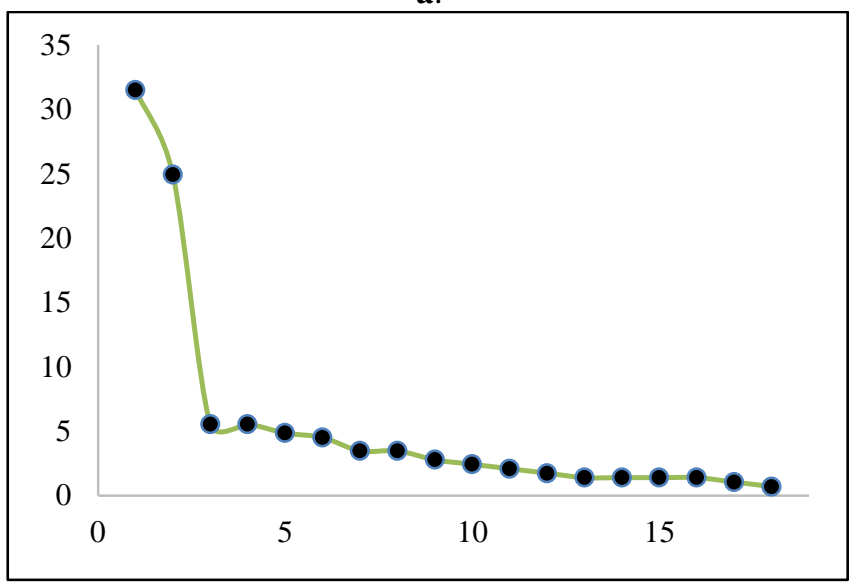

c.

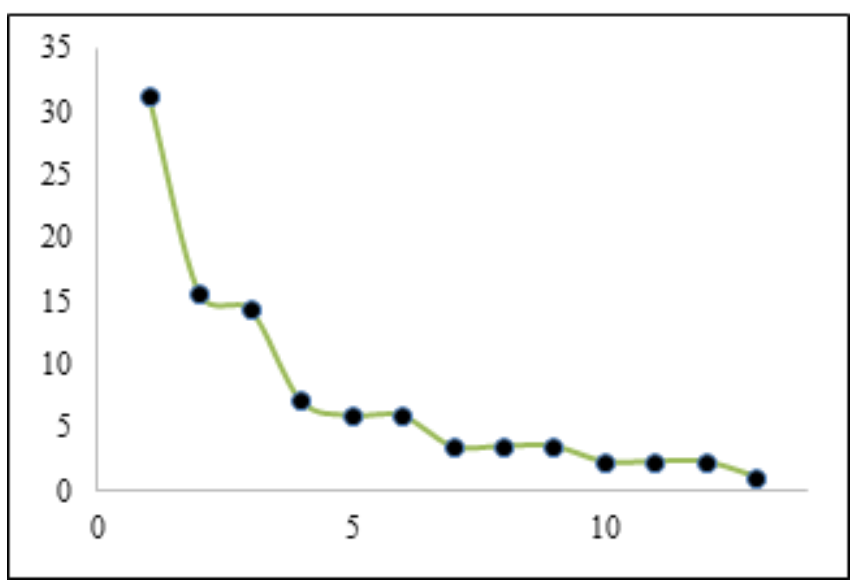

b.

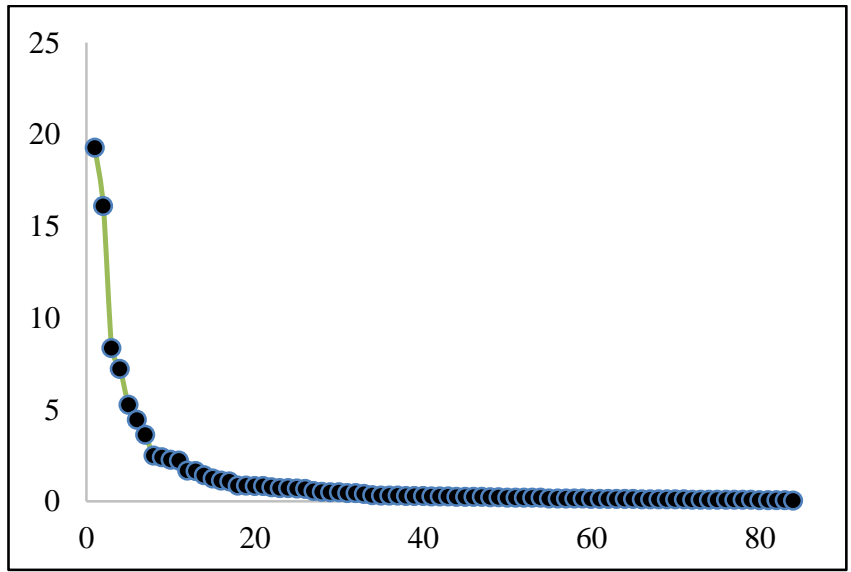

d.

Fig. 5. Rank-abundance curve of the four groups of wildlife where proportion of rank represented vertically and rank of species represented horizontally: a. Amphibia; b. Reptilia; c. Aves; and d. Mammalia.

\section{Mammals}

Among mammals, the relative abundance of lesser bandicoot rat Bandicota indica (31.49\%) was the highest, placing it on the cap of the curve and this is followed by common house rat Funambulus pennantii (24.91\%) (Table 1 and Fig. 5d). Rural habitat provides suitable facilities, such as food sources (e.g. grains, vegetables, fruits, seeds, barks) and make an ideal habitat for living and reproduction thus rising their population in the study area. The conservation and threatened status showed that 117 $(95.12 \%)$ species were least concern, five $(4.06 \%)$ were near threatened and one $(0.81 \%)$ were endangered. Sarker and Sarker (1983) and IUCN (2015) reported that fifteen species of mammals are threatened nationally.

In total 23 species of vertebrate wildlife were rarely seen in the study area (Table 2). Among them seven reptiles, 12 species were birds and four mammals.

Some factors are affecting the wildlife of Sreepur, such as (1) habitat degradation due to the increases of human population, (2) extension of agricultural land and increase of land use in the above mentioned habitats, (3) wide scale destruction of the natural habitats where wild animals are inhabiting, (4) illegal hunting, trapping and collecting of young birds and mammals from their nests causing depletion of wildlife population from the study area, (5) agro-chemicals are being randomly used by the farmers without having proper knowledge, thus adding pollutants to the environment of wildlife and (6) ignorance of local people about the importance of biodiversity and conservation of wildlife species. 
Table 2. Recorded rare vertebrate wildlife in Sreepur upazila, Magura.

\begin{tabular}{|c|c|c|c|c|}
\hline Scientific name & English name & Recorded individuals & Relative Abundance & Density/ km² \\
\hline \multicolumn{5}{|c|}{ Aves (12 species) } \\
\hline Dicrurus aeneus & Bronzed drongo & 3 & 0.08 & 0.11 \\
\hline Psittacula krameri & Rose-ringed parakeet & 3 & 0.08 & 0.11 \\
\hline Saxicola caprata & Pied bushchat & 3 & 0.08 & 0.11 \\
\hline Oriolus oriolus & Eurasian golden oriole & 3 & 0.08 & 0.11 \\
\hline Jynx torquilla & Eurasian wryneck & 3 & 0.08 & 0.11 \\
\hline Phalacrocorax niger & Little cormorant & 3 & 0.08 & 0.11 \\
\hline Centropus bengalensis & Lesser coucal & 3 & 0.08 & 0.11 \\
\hline Tephrodornis pondicerianus & Common woodshrike & 2 & 0.05 & 0.07 \\
\hline Zosterops palpebrosus & Oriental white-eye & 2 & 0.05 & 0.07 \\
\hline Acrocephalus agricola & Paddyfield warbler & 2 & 0.05 & 0.07 \\
\hline Merops philippinus & Blue-tailed bee-eater & 2 & 0.05 & 0.07 \\
\hline Zoothera citrina & Orange-headed trush & 1 & 0.03 & 0.03 \\
\hline \multicolumn{5}{|c|}{ Mammalia (4 species) } \\
\hline Felis chaus & Jungle cat & 4 & 1.38 & 0.14 \\
\hline Vulpes bengalensis & Bengal fox & 4 & 1.38 & 0.14 \\
\hline Canis aureus & Asiatic jackal & 3 & 1.04 & 0.11 \\
\hline Prionailurus viverrinus & Fishing cat & 2 & 0.69 & 0.07 \\
\hline \multicolumn{5}{|c|}{ Reptilia (7 species) } \\
\hline Amphiesma stolatum & Striped keelback & 3 & 3.61 & 1.5 \\
\hline Gekko gecko & Tokay gecko & 3 & 3.61 & 1.5 \\
\hline Naja naja & Binocellate cobra & 2 & 2.41 & 1.0 \\
\hline Ahaetulla nasuta & Vine snake & 2 & 2.41 & 1.0 \\
\hline Typhlops diardii & Diard's blind snake & 2 & 2.41 & 1.0 \\
\hline Ptyas mucosa & Indian rat snake & 1 & 1.20 & 0.5 \\
\hline Varanus bengalensis & Bengal monitor & 3 & 3.61 & 1.5 \\
\hline
\end{tabular}

To protect wildlife diversity, habitat degradation should be prevented. The habitats occupied by the wildlife cannot be hampered by the people. Killing, hunting or trapping of wildlife must be stopped for the survival of threatened (critically endangered, endangered and vulnerable) wildlife. Creation of public awareness through organizing public programs, like discussion in the educational institutions and stakeholders and distribution of leaflets and hand notes may help to increase consciousness about the importance of the wildlife diversity. Besides, preparing and implementing wildlife management plan is important in order to protect the biodiversity in this area.

\section{ACKNOWLEDGMENTS}

We are very much grateful to the local communities and anonymous volunteers for their kind support during the field trips. We are thankful to the honorable Chairman of the Department of Zoology, University of Dhaka for providing the study materials, laboratory facilities and books. We are also thankful to the Ministry of Science and Technology, Bangladesh for their financial support.

\section{REFERENCES}

Al-Razi, H., M. Maria, S. Hasan and S. B. Muzaffar. 2020a. First record of Raorchestes longchuanensis Yang and Li 1978 (Anura: Rhacophoridae) from north-eastern Bangladesh suggests wide habitat tolerance. Amphib. Reptile Conserv. 14(1): 119-131. 
Al-Razi, H., M. Maria and S. B. Muzaffar. 2020b. A new species of cryptic Bush frog (Anura: Rhacophoridae, Raorchestes) from north-eastern Bangladesh. Zoo Keys. 927: 127-151.

Hakim, J., S. J. Trageser, A. Ghose, S. M. A. Rashid and S. C. Rahman. 2020. Amphibians and reptiles from Lawachara National Park in Bangladesh. Check List. 16: 1239-1268.

Halder, R. R. 2010. A photographic guide to the bird of Bangladesh. Baikal Teal Publication, Dhaka, Bangladesh. 257 pp.

Hasan, M. K., M. M. H. Khan and M. M. Feeroz. 2014. Amphibians and Reptiles of Bangladesh: A Field Guide. Arannayk Foundation, Dhaka, Bangladesh. 191 pp.

Hossain, M. L. and S. U. Sarker. 1997. Birds of Hatiya Island, Noakhali, Bangladesh. Dhaka Univ. J. Biol. Sci. 6(1): 39-48.

Hossain, M. L., M. F. Jaman and S. U. Sarkar. 2004. Diversity of Herpeto-Mammalian fauna and their conservation issues in Hatiya Island, Bangladesh. Tropical Biodiv. 8(2): 71-78.

Islam, N., M. F. Jaman, M. M. Rahman and M. M. Alam. 2018. Wildlife Diversity and Population Status of Kashimpur Union, Gazipur, Bangladesh. J. Asiat. Soc. Bangladesh Sci. 44(2): 101-115.

IUCN Bangladesh. 2015. Red List of Bangladesh. Vol. 2-4. Mammals, Birds, Amphibians and Reptiles. IUCN, International Union for Conservation of Nature, Bangladesh Country Office, Dhaka, Bangladesh.

Jaman, M. F., M. F. Rabbe, M. M. Alam, A. R. Shome, M. A. Hossain and M. A. R. Sarker. 2020. Students' perceptions on snake in North-western Bangladesh. Asian J. Ethnobiol. 3(2): 62-69.

Jaman, M. F., M. S. Majumder, M. S. Hossain, M. M. Rahman and M. Uddin. 2014. Diversity of Wildlife at Ruhitpur Union, Keraniganj, Dhaka. J. Asiat. Soc. Bangladesh Sci. 40(2): 295-308.

Jaman, M. F., M. S. Rahman and M. E. Haque. 2011. Diversity of avifauna at the Bangladesh Academy for Rural Development (BARD), Kotbari, Comilla. Univ. J. Zool. Rajshahi Univ. 30: 41-44.

Jaman, M. F., M. Uddin, M. M. Alam, M. M. Rahman, M. T. Khatun and S. M. I. Alam. 2015. Species diversity and population status of wildlife in Keshabpur, Bangladesh. J. biodivers. conserv. bioresour. manag. 1(2): 9-21.

Jaman, M. F., S. U. Sarker and N. J. Sarker. 1999. Food habits and feeding behaviour of black drongo, Dicrurus macrocercus albirictus (Hodgson). Bangladesh J. Zool. 26(2): 57-66.

Jaman, M. F., M. A. R. Sarker, M. M. Alam, M. M. Rahman, M. F. Rabbe, A. S. Rana, A. R. Shome and S. Hossain. 2021. Species diversity, distribution and habitat utilization of urban wildlife in a megacity of Bangladesh. Biodivers. J. 12(3): 635-653.

Kajol, S. B., M. F. Rabbe, M. A. Rahman, A. Asif, K. H. Newaz, M. Sarafat and A. R. Shome. 2020. Range Extension of the Collared Black-headed Snake, Sibynophis collaris (Gray 1853): A New Record from South-eastern Bangladesh. Reptiles \& Amphibians. 27(2): 254-254.

Khan, M. A. R. 2015. Wildlife of Bangladesh-checklist and guide. Chayabithi, Purana Paltan, Dhaka 1000, Bangladesh. 568 pp.

Khan, M. M. H. 2018. A Photographic Guide to Wildlife of Bangladesh. Arannayk Foundation, Dhaka, Bangladesh. 488 pp. 
Mukul, S. A. 2008. Biodiversity Conservation Strategies in Bangladesh: The State of Protected Areas. Tiger Paper. 34(4): 28-32.

Nishat, A., S. M. I. Huq, S. P. Barua, A. H. M. A. Reza and A. S. M. Khan. 2002. Bio-ecological Zones of Bangladesh. IUCN Bangladesh Country Office, Dhaka, Bangladesh. 141 pp.

Rabbi, M. G., S. U. Sarker and M. F. Jaman. 2011. Ecology and status of avifauna of Nijhum Dwip and Damar Char, Noakhali and the conservation issues. J. NOAMI. 28(2): 59-71.

Rahman, H., M. F. Jaman and M. S. Rahman. 2013. Ecology and diversity of wildlife in the eco-park of the Jamuna bridge and its adjacent area, Sirajgonj, Bangladesh. Ecoprint. 20: 27-36.

Rahman, M. S., S. U. Sarker and M. F. Jaman. 2011. Ecological status of the herpeto-mammalian fauna of the Padma river and its adjacent areas, Rajshahi and their conservation issues. J. NOAMI. 28(1): 49-61.

Sarker, N. J., M. F. Jaman, D. Sultana and M. K. Rahman. 2009. Diversity and population of avifauna of two urban sites in Dhaka, Bangladesh. Ecoprint. 16: 1-7.

Sarker, S. U. and N. J. Sarker. 1983. Endangered wildlife of Bangladesh. Tiger Paper. 10(2): 26-28.

Sarker, S. U., M. F. Jaman, M. L. Hossain and N. J. Sarker. 2001. Wildlife diversity of Maheskhali Island: their ecology and conservation issues. J. NOAMI. 18(1): 17-31.

Shome, A. R., M. M. Alam, M. F. Rabbe, Rahman, M. M. and M. F. Jaman. 2020a. Diversity, status and habitat usage of avifauna at Magura Sadar upazila, Bangladesh. Bangladesh J. Zool. 48(2): 441456.

Shome, A. R., M. F. Jaman, M. F. Rabbe, A. I. Barkat and M. M. Alam. 2020b. New distribution record of Rhabdophis subminiatus (Schlegel 1837; Squamata, Colubridae) from Madhupur National Park, Tangail, Bangladesh. Herpetology Notes. 13: 549-551.

Whittaker, R. H. 1965. Dominance and Diversity in Land Plant Communities Numerical relations of species express the importance of competition in community function and evolution. Science. 147: 250-260. 